Published in final edited form as:

Inflamm Bowel Dis. 2015 August ; 21(8): 1976-1981. doi:10.1097/MIB.0000000000000386.

\title{
Iron Replacement in Patients with Inflammatory Bowel Disease - A Systematic Review and Meta-Analysis
}

\author{
Anita Abhyankar, MD and Alan C Moss, MD \\ Center for Inflammatory Bowel Disease, Division of Gastroenterology, Beth Israel Deaconess \\ Medical Center
}

\section{Abstract}

Background-A number of controlled trials and prospective studies have compared intravenous (IV) to oral (PO) iron for the treatment of IDA, with mixed results.

Methods-We conducted a systematic review of trials published to 2014 that compared IV to PO iron to treat iron deficiency in patients with IBD. Meta-analysis was performed to generate effect estimates. Quality assessment was also performed according to GRADE criteria.

Results-Five studies met our inclusion criteria, enrolling 694 patients. For the primary outcome of 'response' ( $\mathrm{Hb}$ rise $>2 \mathrm{~g} / \mathrm{dl}$ ), there was no significant difference between IV or PO iron; risk ratio (RR) for response with IV was 1.08 (95\% CI 0.9, 1.2, p=0.2). For the secondary outcome of mean change in $\mathrm{Hb}(\mathrm{g} / \mathrm{dl})$, the mean difference between $\mathrm{PO}$ and IV iron was not statistically significant (mean difference $0.6 \mathrm{~g} / \mathrm{dL}, 96 \% \mathrm{CI}-0.1,1.3, \mathrm{p}=0.08$ ). IV iron was associated with a significantly greater initial rise in serum ferritin compared to PO iron (mean difference $89 \mathrm{ng} / \mathrm{ml}$, 95\% CI 29, 148, $\mathrm{p}=0.003)$. There was a lower risk of withdrawal due to adverse events in these trials in the IV iron cohorts when compared to PO iron (RR 0.4, 95\% CI 0.1, 1.0, $\mathrm{p}=0.05$ ).

Conclusions-We found no significant difference between IV and PO iron in correcting irondeficiency anemia in patients with IBD in this meta-analysis. Patients who received IV iron had a greater rise in serum ferritin, and were less likely to stop treatment due to adverse events, when compared to those who received PO iron.

\section{Keywords}

IBD; iron deficiency anemia; meta-analysis

Correspondence to: Alan Moss MD, FACG, FEBG, AGAF, Beth Israel Deaconess Medical Center, 330 Brookline Ave, Boston, MA 02215, T: 617-667-3197, F: 617-667-1171, amoss@bidmc.harvard.edu.

Relevant Sources of Support: none

Contributions:

AA - study design, acquisition of data \& analysis, drafting of manuscript.

ACM - study design, analysis \& interpretation of data, statistical analysis, critical review of manuscript, study supervision.

Potential Competing Interests:

None 


\section{INTRODUCTION}

Iron deficiency anemia is an important complication in patients with inflammatory bowel disease (IBD); it is detected in up to $20 \%$ of out-patients and $70 \%$ of in-patients with IBD $(1,2)$. At the patient level, anemia can significantly impair quality of life and productivity, due to the associated fatigue patients experience (3). In addition to fatigue, IBD patients with anemia experience additional complications such as stomatitis and restless legs (4). At an economic level, the costs of care for IBD patients with anemia is more than twice that of non-anemic patients (5).

Although the underlying causes of iron deficiency anemia in IBD patients are usually multifactorial, replacement of depleted iron stores is necessary to avoid side effects and complications of anemia. Replacement of iron stores can be undertaken using oral or intravenous (IV) iron preparations $(6,7)$. Oral iron is convenient and inexpensive, however its efficacy is limited primarily by patient intolerance of adverse effects; up to $21 \%$ of recipients terminate PO iron because of gastrointestinal side effects (7). In contrast, intravenous iron avoids the potential problem of malabsorption and the intestinal side effects of oral preparations. Gisbert (2) reported that the response ( $\mathrm{Hb}$ normalization in 3 months) to IV iron sucrose can be as high as $73 \%$. However, anaphylaxis has been rarely associated with IV iron administration, and the costs associated with IV administration of iron are higher than PO therapy $(2,8)$. In Canada, three infusions of iron sucrose cost $\$ 1831$, compared with $\$ 30$ for 100 tablets of $300 \mathrm{mg}$ PO iron (9). Administration costs of IV therapy in the US would be expected to be even greater. No cost-effectiveness analysis in the U.S. setting has been performed to date.

Given the advantages and disadvantages of both these iron replacement options, a number of studies have compared them directly via controlled trials or open-label studies. Although some have demonstrated superiority for IV iron, others have not $(1,10,11)$. European guidelines (ECCO) have recommended IV replacement as the therapy of choice for IBD patients, based on older RCTs (12). In contrast, the U.S. GI societies have made no recommendations in their guidelines on how to manage anemia in patients with $\operatorname{IBD}(13,14)$. A recent expert panel, using a RAND/UCLA appropriateness method, concluded that highdose IV iron was "more often considered appropriate than other options" for managing anemia in IBD, based on expert opinion (10). Two prior meta-analyses concluded that IV iron was superior to PO for correction of anemia, but both preceded the publication of the recent largest RCT to date $(9,15)$. In light of this uncertainty, we sought to perform a systematic review and meta-analysis of parenteral versus oral iron therapy in IBD patients that includes all relevant studies to 2014 .

\section{METHODS}

\section{Literature search}

A literature search was performed to identify all published and unpublished studies in any language that reported treatment of iron deficiency anemia in patients with IBD. A systematic search of the following database was performed: MEDLINE (Pubmed)-1966 to January 2014, Web of Science-2000 to January 2014), Allied Health Literature 
(CINAHL)-1990 to January 2014, Scopus-2000 to January 2014 and EMBASE 2000January 2014. The following search strategy was constructed by using a combination of MeSH subject headings and text words; 'Inflammatory Bowel Disease," "Crohn's disease," "Ulcerative colitis," "Iron deficiency anemia," "adverse effects", "trial".

\section{Inclusion and Exclusion criteria}

We included prospective controlled trials that compared oral to IV iron in correcting iron deficiency anemia in patients with IBD. The primary outcome measure was a "response", defined as an increase in $\mathrm{Hb}$ of $>2 \mathrm{~g} / \mathrm{dl}$. Secondary outcome measures included mean change in $\mathrm{Hb}$, mean change in serum ferritin, and withdrawal due to adverse events.

We excluded studies if they a) were review articles or b) did not report oral and intravenous $\mathrm{Hb}$ outcomes c) did not investigate IBD patients.

\section{Study selection}

Two authors (AA and AM) independently scanned the abstract of every trial identified by the search to determine eligibility. Blinding to source was not performed. Full articles were selected for further assessment if the abstract suggested that the study included patients with IBD, who were given oral or intravenous iron therapy. If these criteria were unclear from the abstract, the full article was retrieved for clarification. Papers not meeting the inclusion criteria were excluded.

\section{Data Extraction and Quality Assessment}

The following data were retrieved (where possible) from published reports using standardized forms with disagreements resolved by discussion between the reviewers: number of patients in the study, method of selection of patients, numbers of patients with IBD who were given oral or intravenous iron therapy, methods of measurement of clinical outcomes and reporting outcomes used. No distinction was made between different preparations of intravenous or oral iron preparations. A 'risk of bias' assessment and a 'quality' assessment was made for each included study, using standard criteria $(16,17)$.

\section{Statistical Analysis}

Data were analyzed and reported consistent with the consensus guidelines by the Metaanalysis of Observational Studies in Epidemiology group (18). Data were pooled for metaanalysis if the outcomes were sufficiently similar (determined by consensus of authors) and data were homogenous (determined by the degree of clinical and statistical heterogeneity). Data were pooled for meta-analysis for IBD patients treated for iron deficiency anemia with oral or intravenous iron. Raw data from included studies (absolute numbers) were used to construct $2 \times 2$ contingency tables and unadjusted risk ratios (RRs) were calculated using Review Manager (Revman 5.1) for dichotomous outcomes. The random effects model was used to account for variations between studies and give a more conservative pooled estimate (19). The $Q$ test was used to assess for heterogeneity and $\mathrm{I}^{2}$ statistic to quantify the percentage of heterogeneity due to between-study variation; a value of $p<0.10$ was considered statistically significant (20). Funnel plots and the Egger's test were used to evaluate for publication bias (21). Sensitivity analyses were performed for all outcomes 
where 3 or more studies were included. Significance levels were set at a $\mathrm{P}<0.05$. Reporting of this meta-analysis used the checklist recommended by PRISMA (Supplementary Table 1). Additional figures were created using GraphPad Prism (La Jolla, CA).

\section{RESULTS}

\section{Study Characteristics}

We identified 29 studies for full-text review, of which 5 randomized controlled trials met our inclusion criteria (see Fig. 1). Table 1 summarizes the main characteristics of the 5 included studies $(\mathrm{n}=694)(10,11,22,23)$. Twenty-four studies were excluded for reasons detailed in Supplementary Table 2. The overall quality of 4 out of 5 studies was moderate, primarily due to non-blinding of outcome assessors, or incomplete reporting of results (Supplementary Table 3). The results of the 'risk of bias' assessment is provided in Supplementary Figure 1. Funnel plot (Suppl Fig. 2) suggested no publication bias. At baseline, mean $\mathrm{Hb}$ was similar between PO and IV iron cohorts across all studies (Suppl Fig 3A). The mean cumulative dose of IV iron was similar in all studies, but the cumulative doses of PO iron used were highly variable (Suppl Fig 3B).

\section{Outcomes}

A response in $\mathrm{Hb}$ (rise $>2 \mathrm{~g} / \mathrm{dL}$ ) was noted in 143/255 PO iron recipients, and 296/469 IV iron recipients during study follow-up. The risk ratio for response with IV iron was similar to PO iron (RR 1.1, 95\% CI: 0.9-1.2, p=0.2, Fig. 2). There was little statistical heterogeneity in this outcome ( $\mathrm{I}^{2}$ of $8 \%, \mathrm{p}=0.4$ ). Sensitivity analysis for analysis model (fixed or random), year of publication, length of therapy, or baseline $\mathrm{Hb}$ did not change this overall effect estimate. Exclusion of the Erichsen study, which was a cross-over design, and used ferrous fumarate instead of ferrous sulfate, did not change the primary outcome (RR 1.1). Similarly, exclusion of the Reinisch study did not alter the primary outcome (RR 1.2). No adjusted data was reported in the included studies for further subgroup analyses, particularly with regard to IBD disease activity, and PO adherence. Given the broad range of treatment duration used in the included studies, we also plotted effect estimate (RR) from individual studies against duration of therapy (Figure 3A), and total dose of IV iron (Figure 3B) There did not appear to be a relationship between duration of therapy, or total dose of IV iron, and the included studies effect estimates.

The pooled mean difference in $\mathrm{Hb}$ between interventions was $0.6 \mathrm{~g} / \mathrm{dL}$, which was not statistically significant ( $95 \% \mathrm{CI}-0.1$ to $1.3, \mathrm{p}=0.08$, Figure 4$)$. The statistical heterogeneity was high with an $\mathrm{I}^{2}$ of $96 \%$, primarily due to the results of the Reinisch trial (10). This was the only trial where PO iron produced a greater rise in $\mathrm{Hb}$ than IV iron. Exclusion of this trial alters the mean difference in $\mathrm{Hb}$ between groups to a statistically significant $0.8 \mathrm{~g} / \mathrm{dL}$ $(\mathrm{p}=0.001)$, favoring IV iron. Sensitivity analysis for length of therapy also altered the mean $\mathrm{Hb}$ difference between groups, favoring IV iron in trials greater than 12 weeks duration (mean difference $1.2 \mathrm{~g} / \mathrm{dL}, \mathrm{p}<0.001$ ). The relationship between duration of therapy, or total dose of IV iron, and the mean difference in $\mathrm{Hb}$ between interventions when all studies were included is presented in Supplementary Figure 4A \& 4B.

Inflamm Bowel Dis. Author manuscript; available in PMC 2016 August 01. 
The pooled mean difference in serum ferritin between interventions was $89 \mathrm{ng} / \mathrm{ml}$, favoring IV iron (95\% CI 29, 148, $\mathrm{p}=0.003)$. There was significant statistical heterogeneity for this outcome, due to the wide range of differences between individual studies $\left(\mathrm{I}^{2} 97 \%\right)$. There was a non-significant inverse relationship between the mean difference in ferritin in individual studies, and the duration of therapy for each study (data not shown).

Finally, we compared interventions for the risk of withdrawal from treatment allocation due to adverse events. Patients who received IV iron were less likely to discontinue therapy than those who received PO iron (RR 0.4, 95\% CI 0.1, 1.0, p=0.05, Figure 6). There was moderate statistical heterogeneity in this analysis $\left(I^{2} 41 \%\right)$. Sensitivity analysis for analysis model, year of publication, or length of therapy did not change this overall effect estimate.

\section{DISCUSSION}

Commentary on the relative merits of IV iron has become topical recently, with the approval of ferric carboxymaltose injection by the FDA in the U.S. Three RCTs of IV iron sucrose or isomaltoside versus $\mathrm{PO}$ iron reported similar efficacy in improving $\mathrm{Hb}$ levels, but two other RCTs of IV ferric carboxymaltose and iron sucrose concluded that IV iron was superior in correcting anemia $(1,10,11,22,23)$. In light of this evidence, the cumulative expert opinion has been that IV iron is the intervention of choice in patients with $\operatorname{IBD}(8,10)$. In the U.S there are no guidelines from gastroenterology societies on this topic, despite its prevalence in IBD practice. Two prior meta-analyses pre-dated the largest trial published on this topic, and contained only smaller trials $(9,25)$.

This meta-analysis suggests that IV iron is better tolerated, and produces greater elevations in ferritin, and $\mathrm{Hb}$ in most RCTs, than PO iron. However, these physiological advantages did not translate to superiority in correcting anemia in these trials. The pooled mean difference in Hb levels after treatment with IV or PO iron $(0.6 \mathrm{~g} / \mathrm{dL})$ did not lead to a clinically meaningful correction of anemia in these studies (rise in $\mathrm{Hb}>2 \mathrm{~g} / \mathrm{dL}$ ). Whether correction of iron stores, without correction of anemia, impacts clinical outcomes is unknown in IBD.

The main hesitancy to using PO iron in practice has been patient-reported side effects, and concerns about worsening IBD activity. An initial trial of oral iron in Crohn's disease reported that patients on $\mathrm{PO}$ iron had higher CDAI scores than patients on IV iron, suggesting that oral iron had pro-inflammatory properties (22). A closer look at this data, and subsequent comparisons in RCTs, suggests that PO iron may exacerbate GI symptoms in some patients, but not inflammatory indices per se $(2,22,23)$. Minor intestinal upset is common with PO iron, but infrequently leads to discontinuation in studies; only $8 \%$ of patients receiving PO iron in the included studies stopped therapy due to side effects.

IV iron formulations have been limited by the maximum amount of iron delivered per infusion, requiring the costs and inconvenience of multiple infusions. Early iron sucrose preparations had a maximum dose per infusion of $300 \mathrm{mg}$, but newer formulations of carboxymaltose $(750 \mathrm{mg}$ ) and isomaltoside $(1000 \mathrm{mg}$ ) allow higher doses per infusion. In the Reinisch trial most patients only required 1-2 infusions of iron isomaltoside to reach a

Inflamm Bowel Dis. Author manuscript; available in PMC 2016 August 01. 
calculated iron requirement. It has been suggested that one reason for the differences in outcome between IV iron trials is the method used to determine the cumulative dose of IV iron required to restore iron stores (10). Two of the trials in this meta-analysis used a standard dose, and three used the Ganzoni formula to determine iron requirements. It has been suggested that fixed doses of IV iron are more likely to achieve a $\mathrm{Hb}$ response than individualized doses, as some patients may be under-dosed when the Ganzoni formula is used (10,26). This has been described as a limitation of the Reinsich study. However, both trials that reported superiority of IV iron used the Ganzoni formula to determine iron dose $(1,11)$. Exclusion of the Reinisch trial from the primary outcome did not significantly change the effect estimate when IV was compared to PO dosing, suggesting the effects of this large study did not impact the outcome alone. Another explanation for the lack of overall superiority of IV iron in published data is the fact that anemia is multifactorial in patients with IBD, and robust correction of iron stores by themselves may not be sufficient to address all the factors contributing to an individual's anemia.

As with all meta-analyses, there are a number of limitations to the conclusions drawn in this study. We included comparisons of different IV and PO iron formulations and cumulative doses, different enrollment populations, and different lengths of therapy. To assess the effect of this heterogeneity we performed sensitivity analyses and comparisons of the effect estimates across these factors (see Results, and Supplementary Figure 4). There was no significant change in the primary outcome (risk of $\mathrm{Hb}$ rise $>2 \mathrm{~g} / \mathrm{dL}$ ) when these factors were accounted for, strengthening the robustness of the results. The secondary outcome (mean difference in $\mathrm{Hb}$ ) was sensitive to length of therapy, and the inclusion of the Reinisch RCT, which used a different formulation of IV iron to prior studies.

\section{CONCLUSIONS}

There was no significant difference between IV and PO iron in correcting iron-deficiency anemia in patients with IBD in this meta-analysis. Patients who received IV iron had a greater elevation in serum ferritin, and were less likely to stop due to adverse events, when compared to those who received PO iron. Future studies on this topic should optimize the dose of IV iron, and include at least 12 weeks of therapy to determine whether this noninferiority is due to study design.

\section{Supplementary Material}

Refer to Web version on PubMed Central for supplementary material.

\section{Acknowledgments}

Financial Support:

ACM is supported by NIH grant K23DK084338. 


\section{References}

1. Kulnigg S, Stoinov S, Simanenkov V, et al. A novel intravenous iron formulation for treatment of anemia in inflammatory bowel disease: the ferric carboxymaltose (FERINJECT) randomized controlled trial. Am J Gastroenterol. 2008; 103:1182-92. [PubMed: 18371137]

2. Gisbert JP, Gomollón F. Common misconceptions in the diagnosis and management of anemia in inflammatory bowel disease. Am J Gastroenterol. 2008; 103:1299-307. [PubMed: 18477354]

3. Wells CW, Lewis S, Barton JR, et al. Effects of changes in hemoglobin level on quality of life and cognitive function in inflammatory bowel disease patients. Inflamm Bowel Dis. 2006; 12:123-30. [PubMed: 16432377]

4. Weinstock LB, Bosworth BP, Scherl EJ, et al. Crohn's disease is associated with restless legs syndrome. Inflamm Bowel Dis. 2010; 16:275-9. [PubMed: 19575360]

5. Nissenson AR, Wade S, Goodnough T, et al. Economic burden of anemia in an insured population. J Manag Care Pharm. 2005; 11:565-74. [PubMed: 16137214]

6. Stein J, Hartmann F, Dignass AU. Diagnosis and management of iron deficiency anemia in patients with IBD. Nat Rev Gastroenterol Hepatol. 2010; 7:599-610. [PubMed: 20924367]

7. Kulnigg S, Gasche C. Systematic review: managing anaemia in Crohn's disease. Aliment Pharmacol Ther. 2006; 24:1507-23. [PubMed: 17206940]

8. Gasche C, Berstad A, Befrits R, et al. Guidelines on the diagnosis and management of iron deficiency and anemia in inflammatory bowel diseases. Inflamm Bowel Dis. 2007; 13:1545-53. [PubMed: 17985376]

9. Lee TW, Kolber MR, Fedorak RN, et al. Iron replacement therapy in inflammatory bowel disease patients with iron deficiency anemia: a systematic review and meta-analysis. J Crohns Colitis. 2012; 6:267-75. [PubMed: 22405161]

10. Reinisch W, Staun M, Tandon RK, et al. A randomized, open-label, non-inferiority study of intravenous iron isomaltoside 1,000 (Monofer) compared with oral iron for treatment of anemia in IBD (PROCEED). Am J Gastroenterol. 2013; 108:1877-88. [PubMed: 24145678]

11. Lindgren S, Wikman O, Befrits R, et al. Intravenous iron sucrose is superior to oral iron sulphate for correcting anaemia and restoring iron stores in IBD patients: A randomized, controlled, evaluator-blind, multicentre study. Scand J Gastroenterol. 2009; 44:838-45. [PubMed: 19330567]

12. Assche G, Van Dignass A, Reinisch W, et al. The second European evidence-based Consensus on the diagnosis and management of Crohn's disease: Special situations. J Crohns Colitis. 2010; 4:63-101. [PubMed: 21122490]

13. Lichtenstein GR, Hanauer SB, Sandborn WJ. Management of Crohn's disease in adults. Am J Gastroenterol. 2009; 104:465-83. quiz 464, 484. [PubMed: 19174807]

14. Kornbluth A, Sachar DB. Ulcerative colitis practice guidelines in adults: American College Of Gastroenterology, Practice Parameters Committee. Am J Gastroenterol. 2010; 105:501-23. quiz 524. [PubMed: 20068560]

15. Avni T, Bieber A, Steinmetz T, et al. Treatment of anemia in inflammatory bowel diseasesystematic review and meta-analysis. PLoS One. 2013; 8:e75540. [PubMed: 24312441]

16. Balshem H, Helfand M, Schünemann HJ, et al. GRADE guidelines: 3. Rating the quality of evidence. J Clin Epidemiol. 2011; 64:401-6. [PubMed: 21208779]

17. Guyatt GH, Oxman AD, Vist G, et al. GRADE guidelines: 4. Rating the quality of evidence-study limitations (risk of bias). J Clin Epidemiol. 2011; 64:407-15. [PubMed: 21247734]

18. Stroup DF, Berlin JA, Morton SC, et al. Meta-analysis of observational studies in epidemiology: a proposal for reporting. Meta-analysis Of Observational Studies in Epidemiology (MOOSE) group. JAMA. 2000; 283:2008-12. [PubMed: 10789670]

19. DerSimonian R, Laird N. Meta-analysis in clinical trials. Control Clin Trials. 1986; 7:177-88. [PubMed: 3802833]

20. Higgins JPT, Thompson SG. Quantifying heterogeneity in a meta-analysis. Stat Med. 2002; 21:1539-58. [PubMed: 12111919]

21. Egger M, Davey Smith G, Schneider M, et al. Bias in meta-analysis detected by a simple, graphical test. BMJ. 1997; 315:629-34. [PubMed: 9310563]

Inflamm Bowel Dis. Author manuscript; available in PMC 2016 August 01. 
22. Erichsen K, Ulvik RJ, Nysaeter G, et al. Oral ferrous fumarate or intravenous iron sucrose for patients with inflammatory bowel disease. Scand J Gastroenterol. 2005; 40:1058-65. [PubMed: 16165718]

23. Schröder O, Mickisch O, Seidler U, et al. Intravenous iron sucrose versus oral iron supplementation for the treatment of iron deficiency anemia in patients with inflammatory bowel disease-a randomized, controlled, open-label, multicenter study. Am J Gastroenterol. 2005; 100:2503-9. [PubMed: 16279906]

24. Gisbert JP, Bermejo F, Pajares R, et al. Oral and intravenous iron treatment in inflammatory bowel disease: hematological response and quality of life improvement. Inflamm Bowel Dis. 2009; 15:1485-91. [PubMed: 19408339]

25. Avni T, Leibovici L, Gafter-Gvili A. Iron supplementation for the treatment of chronic heart failure and iron deficiency: systematic review and meta-analysis. Eur J Heart Fail. 2012; 14:423-9. [PubMed: 22348897]

26. Evstatiev R, Marteau P, Iqbal T, et al. FERGIcor, a randomized controlled trial on ferric carboxymaltose for iron deficiency anemia in inflammatory bowel disease. Gastroenterology. 2011; 141:846-853.e1-2. [PubMed: 21699794] 


\section{2 screened abstracts}

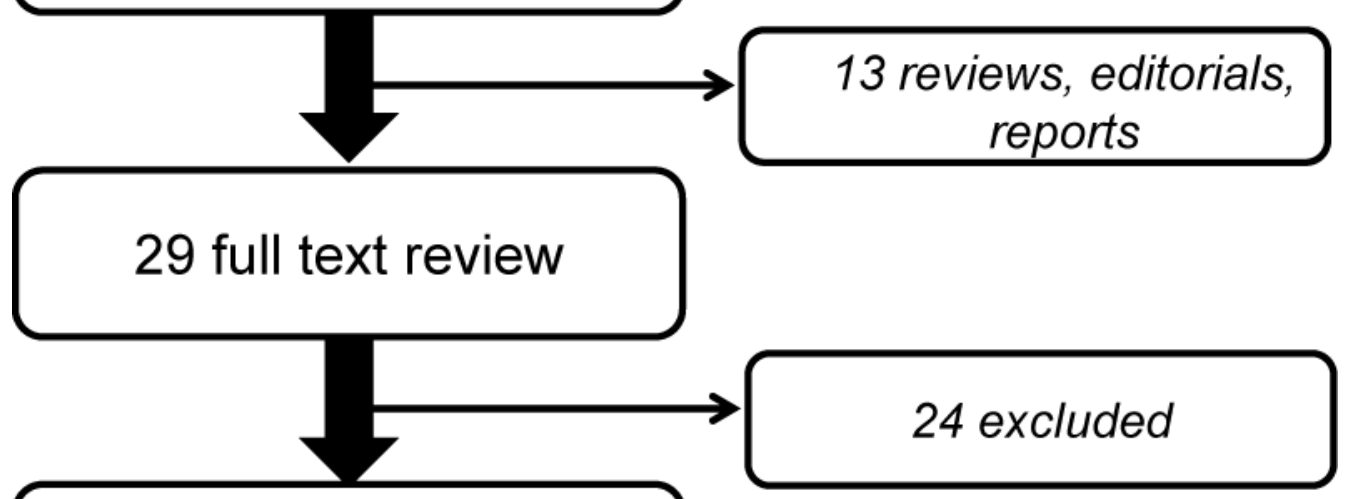

\section{5 included trials}

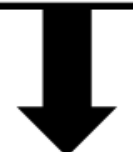

Figure 1.

Flow chart of literature search outcomes 


Study or Subgroup Events Total Events Total Weight M-H, Random, 95\% Cl

Erichsen 2005

Kulnigg 2008

Lindgren 2009

Reinisch 2013

Schroder 2005

$\begin{array}{lllll}3 & 17 & 1 & 17 & 0.4 \%\end{array}$

Random, 95\% Cl

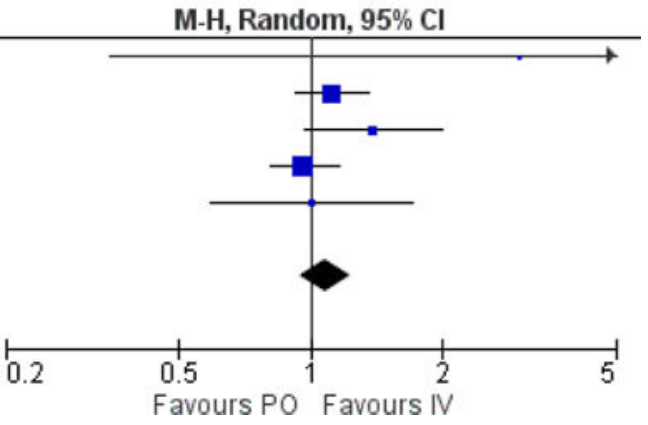

Total $(95 \% \mathrm{Cl})$

$104 \quad 136$

$\begin{array}{lll}41 & 60 & 38.2 \%\end{array}$

$1.12[0.92,1.36$

$1.39[0.97,2.01]$

$\begin{array}{lllll}30 & 45 & 22 & 46 & 12.5 \%\end{array}$

$\begin{array}{lllll}147 & 249 & 66 & 108 & 42.8 \%\end{array}$

$0.97[0.80,1.16]$

$\begin{array}{lllll}12 & 22 & 13 & 24 & 6.1 \%\end{array}$

$1.01[0.59,1.71]$

Total events

469

$255100.0 \%$

$1.08[0.94,1.23]$

Heterogeneity: $\mathrm{Tau}^{2}=0.00 ; \mathrm{Chi}^{2}=4.35, \mathrm{df}=4(P=0.36) ; \mathrm{I}^{2}=8 \%$

Test for overall effect: $Z=1.09(P=0.27)$

Figure 2.

Forest plot of risk ratio of "Response" to iron supplementation (rise in $\mathrm{Hb}<2 \mathrm{~g} / \mathrm{dl}$ ) comparing IV to PO cohorts 
A.

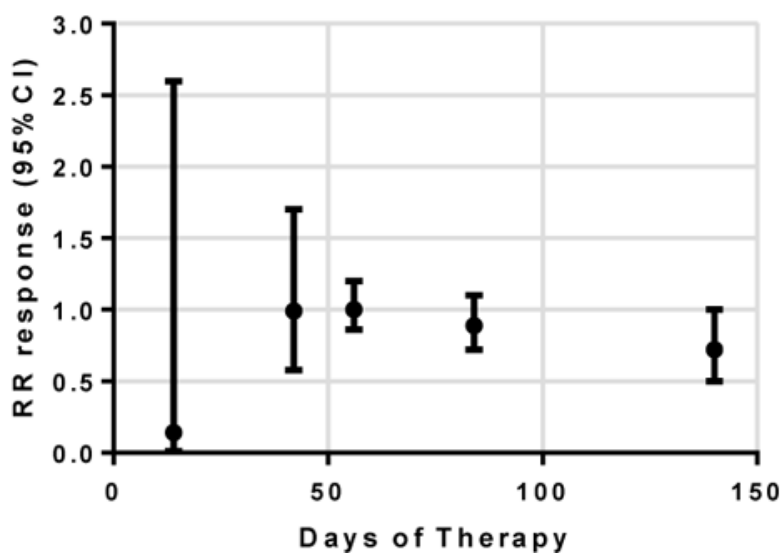

B.

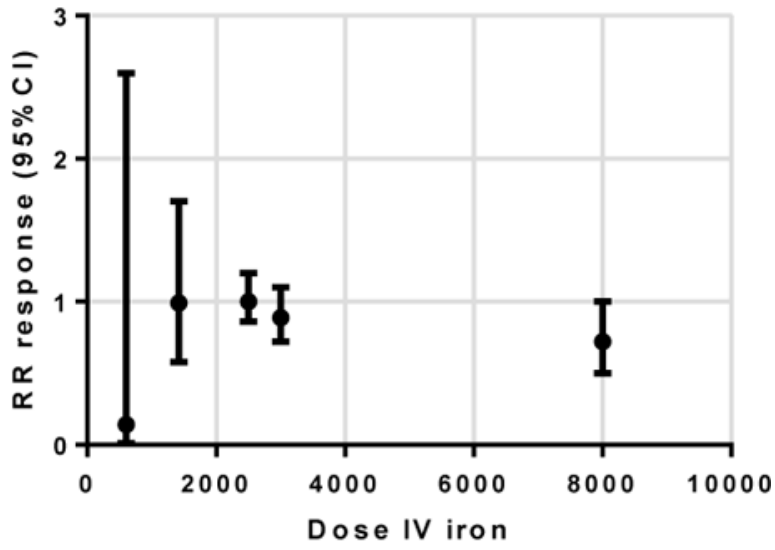

Figure 3.

Plot of risk ratio (and 95\% CIs) for response against duration of therapy (A), and total dose of IV iron (B) 


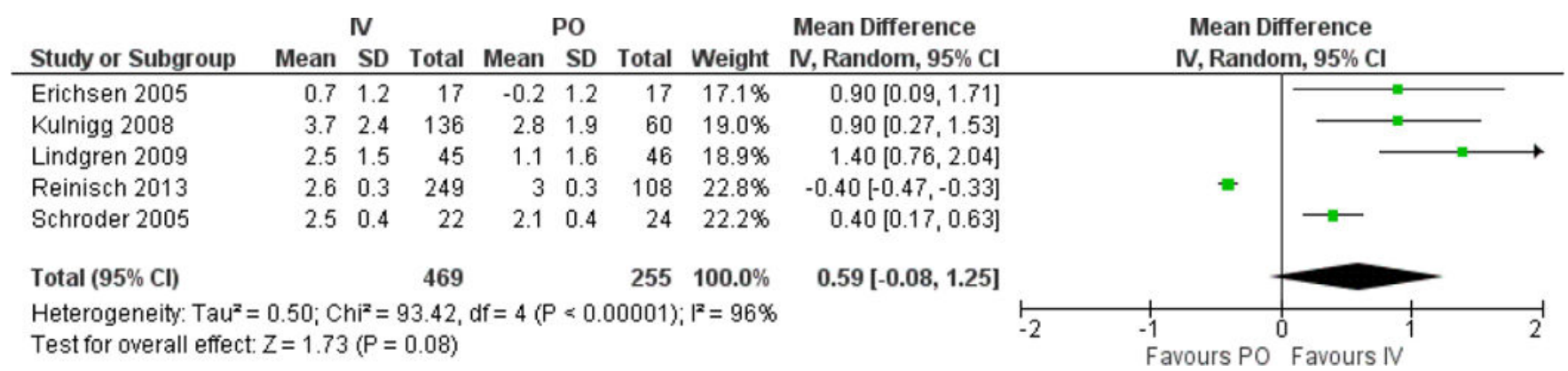

Figure 4.

Forest plot of mean difference in $\mathrm{Hb}$ levels between IV and PO cohorts 


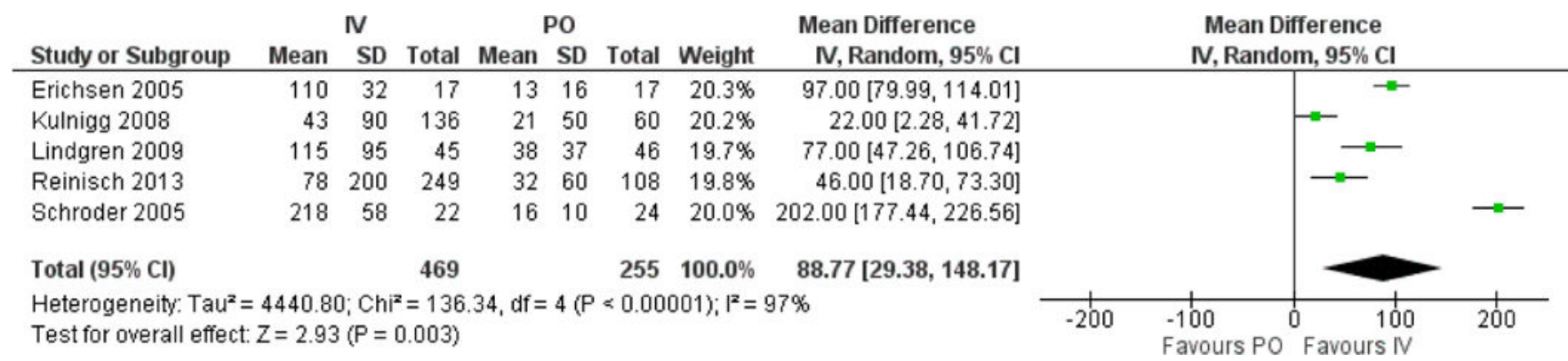

Figure 5.

Forest plot of mean difference in ferritin levels between IV and PO cohorts 


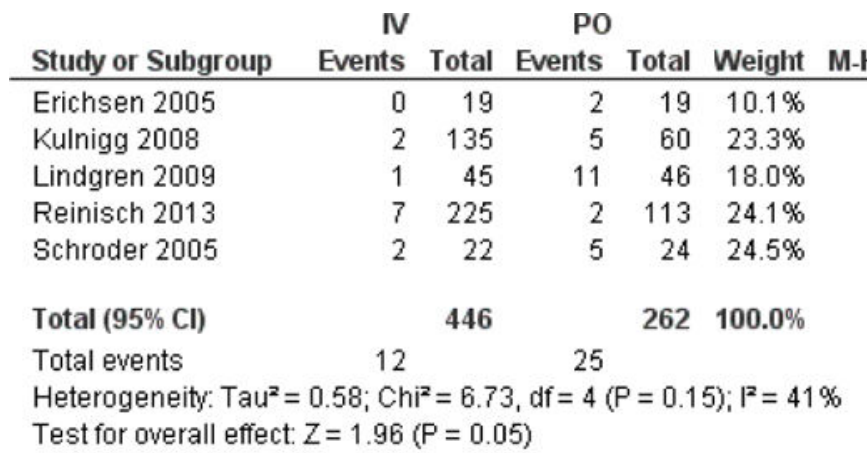

Risk Ratio

Random, $95 \% \mathrm{Cl}$

$0.20[0.01,3.91]$

$0.18[0.04,0.89]$

$0.09[0.01,0.69]$

$1.76[0.37,8.32]$

$0.44[0.09,2.02]$

$0.35[0.12,1.00]$

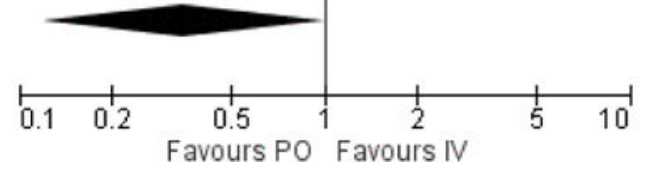

Figure 6.

Forest plot of risk ratio of "withdrawal due to adverse events" comparing IV to PO cohorts 


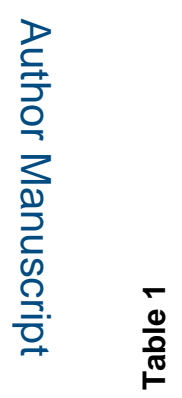

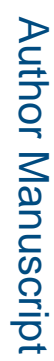

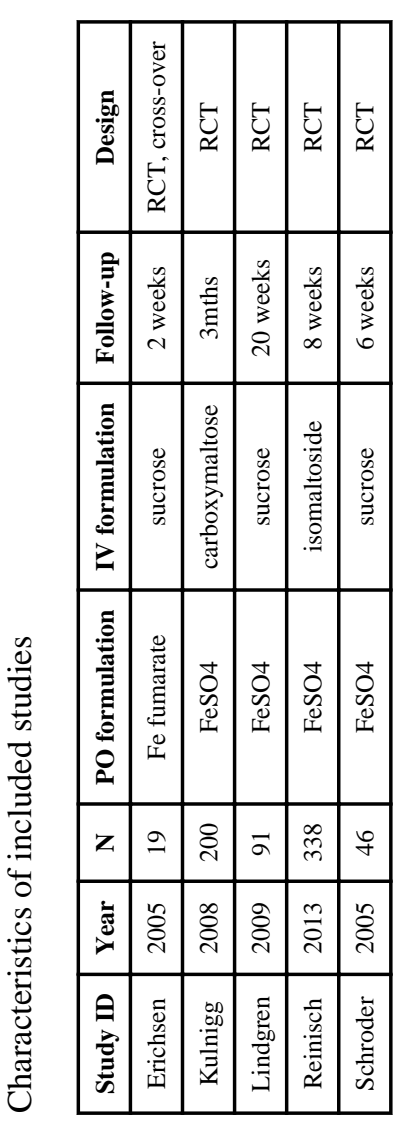

로을

Inflamm Bowel Dis. Author manuscript; available in PMC 2016 August 01. 